\title{
Independent of delay stabilization using implicit Lyapunov function method ${ }^{\star}$
}

\author{
Konstantin Zimenko a $^{\text {, Denis Efimov }}{ }^{\text {a,b,c }}$, Andrey Polyakov ${ }^{\mathrm{a}, \mathrm{b}, \mathrm{c}}$, Artem Kremlev ${ }^{\mathrm{a}}$

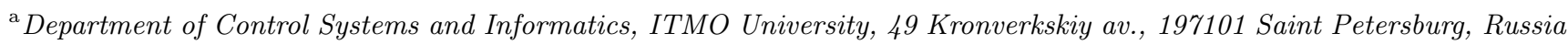 \\ ${ }^{\mathrm{b}}$ Non-A team, INRIA-LNE, Parc Scientifique de la Haute Borne, 40 av. Halley, 59650 Villeneuve d'Ascq, France \\ ${ }^{\mathrm{c}}$ CRIStAL (UMR-CNRS 9189), Ecole Centrale de Lille, BP 48, Cité Scientifique, 59651 Villeneuve-d'Ascq, France
}

\begin{abstract}
Nonlinear implicit Lyapunov function based control algorithms with delay in control and/or measurement channel are presented. The proposed control algorithms provide global asymptotic stability for all delays less than a certain threshold value and global asymptotic stability with respect to a compact set containing the origin for any delay over the threshold value. The algorithms are also applicable for the fast-varying delay and sampled-data cases. The theoretical results are supported by numerical simulations and comparison.
\end{abstract}

\section{Introduction}

Control development for plants with input delays has received increasing attention in the very recent years, due to the wide use of teleoperated and networked systems (see e.g. [2]-[8] to name a few). The lags and delays usually appear in robotic remote control systems, aerospace and seabed applications, medicine (for instance, telesurgery), etc. Such an attention is due to the time-delays can be a source of oscillations and system instability in many cases. Therefore, stability analysis and development of control algorithms, which are robust with respect to uncertain and time-varying delays, are of theoretical and practical importance. Another motivating point for studying this topic is that modern control algorithms are mostly digitally implemented, i.e. in applications sampled-data control naturally appears. Accord-

\footnotetext{
^ The control algorithms presented in Subsection 4.2 were developed under support of RSF (grant 17-19-01422) in ITMO University. The other research were partially supported by ANR 15 CE23 0007 (Project Finite4SoS), the Government of Russian Federation (Grant 08-08) and the Ministry of Education and Science of Russian Federation (Project 14.Z50.31.0031).

Corresponding author K. Zimenko.

${ }^{\star \star}$ The material in this paper was partially presented at the 16th European Control Conference, 2018 [1].

Email addresses: kostyazimenko@gmail.com (Konstantin Zimenko), denis.efimov@inria.fr (Denis Efimov), andrey.polyakov@inria.fr (Andrey Polyakov), kremlev_artem@mail.ru (Artem Kremlev).
}

ing to [13], [5] a sampled-data control system can be represented as a system with time-varying delay in control channel, where the delay is piecewise linear (sawtooth).

Despite of variety of methods solving the described problem, most of them deal with linear control systems. There is a number of control schemes in the literature to handle the systems with input delays: predictive or adaptive control, $H_{\infty}$ control techniques, application of delay identification methods, sliding mode control techniques, etc. (see, for example, [2]-[12], [31], [32] and references inside). Each of these schemes has different features (i.e. whether the delay is known, whether the delay is constant or time-varying, known delay and its derivative bounds, etc.) that should be considered when designing a control system. For example, the use of methods based on Artstein reduction requires knowledge of the delay (or application of delay identification methods [32]), that may suffer from being sensitive to parameter uncertainties and, still more, to delay mismatches [2]. In the case of uncertain delay with known boundaries, the synthesis of control algorithms becomes more complicated. For this case, for most approaches even matching additive disturbances cannot be completely rejected, which implies the ultimate boundedness (in the best case) instead of the asymptotic convergence [2]. For example, the sliding mode control presented in [31] is designed to achieve ultimate boundedness of the closed-loop system with a domain of convergence proportional to the delay and disturbance amplitudes. 
Note, that constructive and computationally tractable conditions (e.g. in the form of Linear Matrix Inequalities (LMIs)) exist for linear time-delay models only [2]. The LMI approach provides constructive finite-dimensional conditions, in spite of significant model uncertainties (see, for example, [5], [31]).

Of particular interest is the use of homogenising control algorithms due to delay robustness properties of nonlinear homogeneous systems. For example, if for zero delay the system is homogeneous with negative degree and globally asymptotically stable, then for any delay it is converging to some compact set around the origin [28]. In other words, the system has delay-independent ISS property, which is not observed for linear systems.

The presented results combine delay robustness properties of nonlinear homogeneous systems with constructive conditions in the LMI form. In particular, the paper addresses robustness analysis of Implicit Lyapunov Function (ILF)-based control system introduced in [29]. It has been shown, that ILF control algorithm preserves boundedness of the system trajectories for any delay and guarantees finite-time stability of the origin in absence of delay. Based on this result in this note ILF-based control algorithms are developed, which preserve asymptotic stability for small delays $0<\tau<\tau_{0}$ and global asymptotic stability with respect to a compact set containing the origin for $\tau \geq \tau_{0}$. Since all proofs in this work do not contain any constraints on the delay derivative, presented algorithms can be used for the case of a time-varying delay $\tau(t)$. Such control algorithms may be useful, for example, when the data transmission channel is unreliable and fickle (for instance, the delay of data transmission depends on the number of network users, there are packet losses, etc.).

The presented feedbacks have several advantages:

- the closed-loop system is robust in the sense of ISS stability with respect to any time-varying delay;

- unlike most of the corresponding approaches, a large increase of the delay value does not lead to an instability of the closed-loop system. Therefore, the proposed results can be used for system protection against unforeseen increase of the delay;

- the feedbacks allow to reject some non-Lipschitz disturbances;

- the results don't require the use of delay identification algorithms;

- in the absence of delay one of the presented feedbacks provides finite-time stability.

Notation: $\mathbb{R}^{n}$ denotes the $n$ dimensional Euclidean space with vector norm $|\cdot|, \mathbb{R}_{>}=\{s \in \mathbb{R}: s>0\}, \mathbb{R}_{\geq}=$ $\{s \in \mathbb{R}: s \geq 0\}, \operatorname{diag}\left\{\lambda_{i}\right\}_{i=1}^{n}$ is the diagonal matrix with the elements $\lambda_{i}$ on the main diagonal, the notation $P>0$, for $P \in \mathbb{R}^{n \times n}$ means that $P$ is symmetric and positive definite, the minimal and maximal eigenvalues of $P$ are denoted by $\lambda_{\min }(P)$ and $\lambda_{\max }(P)$, respectively. The Banach space of continuous functions $\phi:[-\tau, 0] \rightarrow$ $\mathbb{R}^{n}$ with the uniform norm $\|\phi\|=\sup _{-\tau \leq \varsigma \leq 0}|\phi(\varsigma)|$ is denoted by $C_{[-\tau, 0]}$. The symbol $\overline{1, m}$ is used to denote a sequence of integers $1, \ldots, m$.

\section{Problem formulation}

Consider a single input control system of the form

$$
\dot{x}(t)=A x(t)+b u(t)+d(t, x(t))
$$

where $x(t) \in \mathbb{R}^{n}$ is the state vector, $u(t) \in \mathbb{R}$ is the control input, $A=\left(\begin{array}{ccccc}0 & 1 & 0 & \cdots & 0 \\ 0 & 0 & 1 & \cdots & 0 \\ \vdots & \vdots & \vdots & \ddots & \ddots \\ 0 & 0 & \vdots & \vdots \\ 0 & 0 & 0 & \cdots & 1 \\ 0 & 0 & \cdots & \cdots & 0\end{array}\right), b=\left(\begin{array}{c}0 \\ 0 \\ \vdots \\ 0 \\ 1\end{array}\right), d: \mathbb{R}^{n+1} \rightarrow$ $\mathbb{R}^{n}$ describes the system nonlinearities, uncertainties and disturbances, which may be non-Lipschitz or discontinuous. The presented system appears for a number of control applications since many controllable single-input nonlinear systems can be transformed to a canonical form like (1) (see, for example, [14]). Note that in the presence of delayed control the use of feedback transformations leads to appearance of additional terms that should be considered as disturbances.

Assume that there is delay $\tau$ in the control (and/or measurement) channel, i.e.

$$
\dot{x}=A x(t)+b u(t-\tau)+d(t, x),
$$

or retreating $\int_{t-\tau}^{t} \dot{u}(s) d s$ as a disturbance

$$
\dot{x}=A x(t)+b u(t)+d(t, x)+d_{\tau}(t),
$$

where $d_{\tau}(t)=-b \int_{t-\tau}^{t} \dot{u}(s) d s$. It is assumed that the delay $\tau$ is unknown, but its upper bound is known, i.e. $\tau<\tau_{0}, \tau_{0} \in \mathbb{R}_{>}$.

The aim of the paper is to develop the stabilizing control algorithms for the system (2), which

- provide finite-time attractiveness of some compact set around the origin for any delay $\tau$ (for $\tau=0$ the origin is finite-time stable);

- provide asymptotic stability of the system for the delay $\tau<\tau_{0}, \tau_{0} \in \mathbb{R}_{>}$and preserve boundedness of the system trajectories for any delay $\tau \geq \tau_{0}$.

\section{Preliminaries}

Let us consider the system

$$
\dot{x}=f(x), \quad x(0)=x_{0},
$$


where $x \in \mathbb{R}^{n}$ is the state vector, $f \in R^{n} \rightarrow \mathbb{R}^{n}$ is a vector field. If the vector field $f$ is discontinuous with respect to $x$, then the solutions of (3) fall into the area of differential inclusions and need to be understood in the sense of Filippov [15].

\subsection{Implicit Lyapunov function method}

The following theorem presents the ILF method [22], [23] for finite-time stability analysis.

Theorem 1 [29] If there exists a continuous function

$$
\begin{aligned}
& Q: \mathbb{R}_{>} \times \mathbb{R}^{n} \rightarrow \mathbb{R} \\
& (V, x) \mapsto Q(V, x)
\end{aligned}
$$

such that

C1) $Q(V, x)$ is continuously differentiable for $\forall x \in$ $\mathbb{R}^{n} \backslash\{0\}$ and $\forall V \in \mathbb{R}_{>}$;

C2) for any $x \in \mathbb{R}^{n} \backslash\{0\}$ there exist $V^{-} \in \mathbb{R}_{>}$and $V^{+} \in \mathbb{R}_{>}$such that $Q\left(V^{-}, x\right)<0<Q\left(V^{+}, x\right)$;

C3) for $\Omega=\left\{(V, x) \in \mathbb{R}^{n+1}: Q(V, x)=0\right\}$

$$
\lim _{\substack{x \rightarrow 0 \\(V, x) \in \Omega}} V=0, \lim _{\substack{V \rightarrow 0^{+} \\(V, x) \in \Omega}}\|x\|=0, \lim _{\substack{\|x\| \rightarrow \infty \\(V, x) \in \Omega}} V=+\infty ;
$$

C4) the inequality $-\infty<\frac{\partial Q(V, x)}{\partial V}<0$ holds for $\forall V \in \mathbb{R}_{>}$ and $\forall x \in \mathbb{R}^{n} \backslash\{0\}$;

C5) the inequality $\frac{\partial Q(V, x)}{\partial x} f(x) \leq h V^{1-\mu} \frac{\partial Q(V, x)}{\partial V}$ holds $\forall(V, x) \in \Omega$, where $0<\mu \leq 1$ and $h>0$ are some constants.

Then the origin of the system (3) is globally finite-time stable with the settling time estimate $T\left(x_{0}\right) \leq \frac{V_{0}^{\mu}}{h \mu}$.

Based on this result in [29] a finite-time stabilizing control law has been developed for the system (1).

Introduce the implicitly defined Lyapunov function by

$$
Q(V, x)=x^{T} D\left(V^{-1}\right) P D\left(V^{-1}\right) x-1,
$$

where $D(\lambda)$ is the diagonal matrix of the form $D(\lambda)=$ $\operatorname{diag}\left\{\lambda^{1+(n-1) \mu}\right\}_{i=1}^{n}$ and $P \in \mathbb{R}^{n \times n}: P=P^{T}>0$. Denote the diagonal matrix $H_{\mu}=\operatorname{diag}\{1+(n-i) \mu\}_{i=1}^{n}$.

Theorem 2 [29], [19] If

- the system of matrix inequalities

$$
\left\{\begin{array}{l}
A X+X A^{T}+b y+y^{T} b^{T}+L+R \leq 0 \\
\frac{1}{\alpha} L \geq X H_{\mu}+H_{\mu} X>0, \quad X>0, \quad R>0
\end{array}\right.
$$

is feasible for some $R \in \mathbb{R}^{n \times n}, \mu \in(0,1], \alpha \in \mathbb{R}_{>}$, $X \in \mathbb{R}^{n \times n}$ and $y \in \mathbb{R}^{1 \times n}$;
- the control has the form

$$
u(V, x)=V^{1-\mu} k D\left(V^{-1}\right) x,
$$

where $k=y X^{-1}$,

$$
V \in \mathbb{R}_{>}: Q(V, x)=0
$$

and $Q(V, x)$ is defined by (4) with $P=X^{-1}$;

- the disturbance function $d(t, x)$ satisfy

$$
\begin{gathered}
d^{T}(t, x) D\left(V^{-1}\right) R^{-1} D\left(V^{-1}\right) d(t, x) \leq \\
\beta V^{-2 \mu} x^{T} D\left(V^{-1}\right) P L P D\left(V^{-1}\right) x
\end{gathered}
$$

with $\beta \in(0,1)$ and $(t, x) \in \mathbb{R}_{>} \times \mathbb{R}^{n}$.

Then the closed-loop system (1), (6) is globally finitetime stable and the settling time function estimate has the form $T\left(x_{0}\right) \leq \frac{V_{0}^{\mu}}{\alpha \mu(1-\beta)}$, where $V_{0} \in \mathbb{R}_{>}: Q\left(V_{0}, x_{0}\right)=0$.

\subsection{Weighted homogeneity}

For any $r_{i}>0, i=\overline{1, n}$ and $\lambda>0$, define the dilation matrix $D(\lambda)=\operatorname{diag}\left\{\lambda^{r_{i}}\right\}_{i=1}^{n}$ and the vector of weights $r=\left(r_{1}, \ldots, r_{n}\right)^{T}$.

For any $r_{i}>0, i=\overline{1, n}$ and $x \in \mathbb{R}^{n}$ the homogeneous norm can be defined as follows [25], [16]

$$
|x|_{r}=\left(\sum_{i=1}^{n}\left|x_{i}\right|^{\rho / r_{i}}\right)^{1 / \rho}, \rho \geq \max _{1 \leq i \leq n} r_{i}
$$

For any $r_{i}>0, i=\overline{1, n}$ and $\phi \in C_{[-\tau, 0]}$ the homogeneous norm can be defined as follows [21]

$$
\|\phi\|_{r}=\left(\sum_{i=1}^{n}\left\|\phi_{i}\right\|^{\rho / r_{i}}\right)^{1 / \rho}, \rho \geq \max _{1 \leq i \leq n} r_{i} .
$$

Define $B_{\rho}^{\tau}=\left\{\phi \in C_{[-\tau, 0]}:\|\phi\|_{r} \leq \rho\right\}$ as a closed ball of radius $\rho>0$ in $C_{[-\tau, 0]}$.

Consider an autonomous functional differential equation of retarded type [27]:

$$
\dot{x}(t)=f\left(x_{t}\right), t \geq 0,
$$

where $x(t) \in \mathbb{R}^{n}$ and $x_{t} \in C_{[-\tau, 0]}$ is the state function, $x_{t}(s)=x(t+s)$ for $s \in[-\tau, 0], f: C_{[-\tau, 0]} \rightarrow \mathbb{R}^{n}$ is continuous and ensures existence and uniqueness of solutions in forward time [27], $f(0)=0$. We assume that the system (9) for the initial functional condition $x_{0} \in C_{[-\tau, 0]}$ has a unique solution. 
Definition 5 [24] The function $g: C_{[-\tau, 0]} \rightarrow \mathbb{R}$ (vector field $\left.f: C_{[-\tau, 0]} \rightarrow \mathbb{R}^{n}\right)$ is called $r$-homogeneous $\left(r_{i}>0\right.$, $i=\overline{1, n}$ ), if for any $\phi \in C_{[-\tau, 0]}$ the relation

$$
\begin{aligned}
& g(D(\lambda) \phi)=\lambda^{d} g(\phi) \\
& \left(f(D(\lambda) \phi)=\lambda^{d} D(\lambda) f(\phi)\right)
\end{aligned}
$$

holds for some $d \in \mathbb{R}\left(d \geq-\min _{1 \leq i \leq n} r_{i}\right)$ and all $\lambda>0$.

In both cases, the constant $d$ is called the degree of homogeneity. The system (9) is called $r$-homogeneous of degree $d$ if the function $f$ admits this property.

The introduced notion of weighted homogeneity in $C_{[-\tau, 0]}$ is reduced to the standard one in $\mathbb{R}^{n}$ if $\tau=0$.

Theorem 3 [25] Let $f: \mathbb{R}^{n} \rightarrow \mathbb{R}^{n}$ be defined on $\mathbb{R}^{n}$ and be a continuous $r$-homogeneous vector field with degree d $(d<0)$. If the origin of the system (3) is locally asymptotically stable then it is globally asymptotically stable (globally finite-time stable) and there exists a continuously differentiable Lyapunov function $V$ which is $r$-homogeneous of degree $v>-d$.

By definition of homogeneity there exist constants $c_{1}, c_{2}>0$ such that

$$
c_{1}\|x\|_{r}^{v} \leq V(x) \leq c_{2}\|x\|_{r}^{v} .
$$

For example, the system (1), (6) for $d(t, x)=0$ is $r$ homogeneous of degree $-\mu$ with $r=(1+(n-1) \mu, 1+$ $(n-2) \mu, \ldots, 1)^{T}$ and the ILF is homogeneous of degree 1 , where the parameters $c_{1}, c_{2}$ can be estimated as follows

$$
\begin{aligned}
& c_{1}=\min \left\{\frac{\lambda_{\min }^{\frac{1}{2 r_{\max }}}(P)}{n^{\frac{1}{\rho}}}, \frac{\lambda_{\min }^{\frac{1}{2 r_{\min }}}(P)}{n^{\frac{1}{\rho}}}\right\}, \\
& c_{2}=\max \left\{\left(n \lambda_{\max }(P)\right)^{\frac{1}{2 r_{\max }}},\left(n \lambda_{\max }(P)\right)^{\frac{1}{2 r_{\min }}}\right\} \text {. }
\end{aligned}
$$

In [26], [28], [17], [18] robustness with respect to delays has been discussed for homogeneous systems.

Lemma 1 [26] Let $f\left(x_{\tau}\right)=F[x(t), x(t-\tau)]$ in (9) and the system (9) be $r$-homogeneous with degree $d \geq 0$ and globally asymptotically stable at the origin for $\tau=0$, then for any $\rho>0$ there is $0<\tau_{0}<+\infty$ such that (9) is asymptotically stable at the origin in $B_{\rho}^{\tau}$ for any delay $0 \leq \tau \leq \tau_{0}$.

Lemma 2 [28] Let $f\left(x_{t}\right)=F[x(t), x(t-\tau)]$ in (9) be uniformly continuous and the system (9) be $r$ homogeneous with degree $d<0$ and globally asymptotically stable at the origin for $\tau=0$, then for any $\varepsilon>0$ there is $0<\tau_{0}<+\infty$ such that (9) is globally asymptotically stable with respect to $B_{\varepsilon}^{\tau}$ for any delay $0 \leq \tau \leq \tau_{0}$.

\section{Main results}

In this section, first, we provide an LMI-based approach to evaluate the domain of attraction of a finite-time stable control (6) in the case of the delayed plant in (2). Second, a uniting control is designed with commutation between two laws providing a global bounded of all trajectories in (2) for any delay, and convergence to the origin for a sufficiently small one.

4.1 Control providing boundedness of the system trajectories in presence of delay

Lemma 3 (Disturbance-free case) Let the system of linear matrix inequalities

$$
\left\{\begin{array}{l}
A X+X A^{T}+b y+y^{T} b^{T}+L+R \leq 0, \\
\iota X \geq A X+X A^{T} \geq-\iota X, \quad X>0, \quad L \geq \varpi X, \\
X H_{\mu}+H_{\mu} X \geq \eta X, \quad\left(\begin{array}{ll}
X & y^{T} \\
y & \zeta
\end{array}\right) \geq 0,
\end{array}\right.
$$

be feasible for $R=\left(\begin{array}{cc}0_{n-1} & 0 \\ 0 & r_{n n}\end{array}\right)$ and some $\mu \in(0,1]$, $r_{n n}, \eta, \iota, \zeta, \varpi \in \mathbb{R}_{>}, X \in \mathbb{R}^{n \times n}, y \in \mathbb{R}^{1 \times n}$. The system (2), (6) with $d(t, x)=0$ is globally asymptotically stable with respect to $B_{c / c_{1}}^{2 \tau}$ for any $c \in \mathbb{R}_{>}$if

$$
\tau<\frac{\sqrt{r_{n n} \varpi} c^{\mu}}{\eta^{-1} \sqrt{l_{1}}\left(\iota+2 \sqrt{P_{n n} \zeta}\right)+\sqrt{l_{2}}+\left|k_{n}\right| \sqrt{\zeta}},
$$

where $P=X^{-1}, k=y X^{-1}, l_{1}=\lambda_{\max }\left(X^{1 / 2}((1-\mu) I+\right.$ $\left.\left.H_{\mu}\right) k^{T} k\left((1-\mu) I+H_{\mu}\right) X^{1 / 2}\right), l_{2}=\lambda_{\max }\left(X^{1 / 2} A^{T} k^{T} k A X^{1 / 2}\right)$.

Note that in absence of the delay $(\tau=0)$ the origin is finite-time stable.

Proof. The system (2), (6) for $d(t, x(t))=0$ is $r$ homogeneous of degree $-\mu$ and according to [28] all solutions of (2) stay bounded for $0 \leq t \leq \tau$ and converge to some compact set around the origin asymptotically.

In accordance with Theorem 2 one can obtain $\dot{V}(x(t)) \leq$ $-C V^{1-\mu}(x(t)), C \in \mathbb{R}_{>}$if the following inequality holds

$$
\begin{aligned}
& d_{\tau}^{T}(t) D\left(V^{-1}(t)\right) R^{-1} D\left(V^{-1}(t)\right) d_{\tau}(t) \leq \\
& \beta V^{-2 \mu}(t) x^{T}(t) D\left(V^{-1}(t)\right) P L P D\left(V^{-1}(t)\right) x(t)
\end{aligned}
$$

for some $\beta \in(0,1)$. Rewriting this inequality after substitution $d_{\tau}(t)=b \int_{t-\tau}^{t} \dot{u}(s) d s$ we yield

$$
\left(\int_{t-\tau}^{t} \dot{u}(s) d s\right)^{2} \leq r_{n n} \beta \varpi V(t)^{2-2 \mu} .
$$


Now, let us consider derivative of the ILF (7) calculated for the system (2) for $t \geq \tau$. Denote $V(t)=V(x(t))$. Firstly, introduce $\gamma=1+\varrho$ for some $\varrho>0$ and following LyapunovRazumikhin approach consider the term $\dot{V}(t)$ for the case $\gamma V(t) \geq V(t+\theta), \forall \theta \in[-2 \tau, 0]$. According to [29] we obtain

$$
\begin{aligned}
& |\dot{V}(x(t))|= \\
& \mid\left(x^{T}(t) D\left(V^{-1}(t)\right)\left(H_{\mu} P+P H_{\mu}\right) D\left(V^{-1}(t)\right) x(t)\right)^{-1} \times \\
& \left(V^{1-\mu}(t) x^{T}(t) D\left(V^{-1}(t)\right)\left(P A+A^{T} P\right) D\left(V^{-1}(t)\right) x(t)+\right. \\
& \left.2 V^{1-\mu}(t-\tau) x^{T}(t) D\left(V^{-1}(t)\right) P b k D\left(V^{-1}(t-\tau)\right) x(t-\tau)\right) \mid \leq \\
& \eta^{-1} V^{1-\mu}(t) \times \\
& \left(\left|x^{T}(t) D\left(V^{-1}(t)\right)\left(P A+A^{T} P\right) D\left(V^{-1}(t)\right) x(t)\right|+\right. \\
& \left.\left|2 \gamma^{1-\mu} x^{T}(t) D\left(V^{-1}(t)\right) P b k D\left(V^{-1}(t-\tau)\right) x(t-\tau)\right|\right) \leq \\
& \eta^{-1} V(t)^{1-\mu}\left(\iota+2 \gamma^{1-\mu} \sqrt{P_{n n} \zeta}\right) .
\end{aligned}
$$

In order to apply Lyapunov-Razumikhin Theorem assume that $\gamma V(t) \geq \max \left\{c, \sup _{\theta \in[-2 \tau, 0]} V(t-\theta)\right\}$ for $c \in \mathbb{R}_{>}$, then considering the term $\int_{t-\tau}^{t} \dot{u}(s) d s$ with $V(t) \geq \frac{c}{\gamma}$ and taking into account (15) we obtain

$$
\begin{aligned}
& \left|\int_{t-\tau}^{t} \dot{u}(s) d s\right|= \\
& \int_{t-\tau}^{t} V^{-\mu}(s) \dot{V}(s) k\left((1-\mu) I+H_{\mu}\right) D\left(V^{-1}(s)\right) x(s) d s+ \\
& \int_{t-\tau}^{t} V^{1-2 \mu}(s) k A D\left(V^{-1}(s)\right) x(s)+ \\
& \int_{t-\tau}^{t} V^{-\mu}(s) V^{1-\mu}(s-\tau) k b k D\left(V^{-1}(s-\tau)\right) x(s-\tau) d s \mid \leq \\
& \eta^{-1}\left(\iota+2 \gamma^{1-\mu} \sqrt{P_{n n} \zeta}\right) \sqrt{l_{1}}\left|\int_{t-\tau}^{t} V^{1-2 \mu}(s) d s\right|+ \\
& \sqrt{l_{2}}\left|\int_{t-\tau}^{t} V^{1-2 \mu}(s) d s\right|+\gamma^{1-\mu}\left|k_{n}\right| \sqrt{\zeta}\left|\int_{t-\tau}^{t} V^{1-2 \mu}(s) d s\right| \leq \\
& \left(\eta^{-1} \sqrt{l_{1}}\left(\iota+2 \gamma^{1-\mu} \sqrt{P_{n n} \zeta}\right)+\sqrt{l_{2}}+\gamma^{1-\mu}\left|k_{n}\right| \sqrt{\zeta}\right) \times \\
& \tau c^{-\mu} \gamma^{1-\mu} V^{1-\mu}(t) \text {. }
\end{aligned}
$$

Substituting (16) into (14) we yield

$$
\tau \leq \frac{\sqrt{r_{n n} \beta \varpi} c^{\mu}}{\gamma^{1-\mu}\left(\eta^{-1} \sqrt{l_{1}}\left(\iota+2 \gamma^{1-\mu} \sqrt{P_{n n} \zeta}\right)+\sqrt{l_{2}}+\gamma^{1-\mu}\left|k_{n}\right| \sqrt{\zeta}\right)} .
$$

Since $\varrho$ can be chosen arbitrary small and $\beta$ can be chosen arbitrary close to 1 we can rewrite this expression as strict inequality (13). Thus, according to Lyapunov-Razumikhin Theorem [20] the system is asymptotically stable with respect to the set $\Omega_{c}=\left\{x \in C_{[-2 \tau, 0]}: V(x(s)) \leq c, s \in[-2 \tau, 0]\right\}$. Finally, since $\Omega_{c} \subset B_{c / c_{1}}^{2 \tau}$ validity of Lemma 3 is thus concluded.

Remark 1 From the results presented in Lemma 3, knowing the value of delay, one can find the compact set $B_{c / c_{1}}^{2 \tau}$ the system asymptotically converging to. And vise versa, for a given $c$ one can easily find a value $\tau_{0}$

such that for any $\tau<\tau_{0}$ the system (2), (6) is globally asymptotically stable with respect to $B_{c / c_{1}}^{2 \tau}$.

Remark 2 The result of Lemma 3 holds for the case of time-varying delay and sampled-data control realization.

Remarks 1, 2 remain true for the all subsequent results presented below.

Corollary 1 The system (2), (6) can be asymptotically stabilized with respect to an arbitrary small set for any bounded delay by appropriate choosing the parameter $c$ and changing the matrix variable $\tilde{P}=P / v, v \in \mathbb{R}_{>}$.

Proof. Let the LMI (12) is feasible. Then for the change of the matrix variable $\tilde{P}=P / v, v \in \mathbb{R}_{>}$we can obtain the corresponding result with $\tilde{X}=\tilde{P}^{-1}=v X$, $\tilde{y}=v y, \tilde{k}=\tilde{y} \tilde{X}^{-1}=k, \tilde{R}=v R$ and $\tilde{\iota}=\iota, \tilde{\varpi}=\varpi$, $\tilde{\eta}=\eta, \tilde{\zeta}=v \zeta, \tilde{l}_{1}=v l_{1}$ and $\tilde{l}_{2}=v l_{2}$. Note that this change does not affect on the condition (13) and the system is asymptotically stable with respect to the set $\Omega_{c}$. According to (4) with $\tilde{P}=P / v$ we have $\Omega_{c} \subset \Gamma=\left\{x \in C_{[-2 \tau, 0]}:\|x\| \leq \frac{\sqrt{v} \max \left\{c^{\left.r_{\min }, c^{r_{\max }}\right\}}\right.}{\sqrt{\lambda_{\min }(P)}}\right\}$. Then by choosing the parameters $v$ and $c$ the system can be asymptotically stabilized with respect to an arbitrary small set $\Gamma$ and any delay $\tau$ (i.e. the bigger value $c$ the bigger $\tau_{0}$ and the smaller value $v$ the smaller set $\Gamma$ ).

Example 1 Consider the system (2) for $n=3$ in the disturbance-free case $(d(t, x(t))=0)$. Define ILFbased control in the form (6) with the parameter $\mu=0.3$. The matrix $P>0$ and the vector $k$ are obtained from the system (12) with $r_{n n}=0.99, \eta=1$ : $P=\left(\begin{array}{lll}1.0191 & 2.0359 & 1.0155 \\ 2.0359 & 5.0857 & 3.0452 \\ 1.0155 & 3.0452 & 3.0446\end{array}\right)$ and $k=\left(\begin{array}{l}-1.0046 \\ -3.0112 \\ -3.0074\end{array}\right)^{T}$.

Obtained value of maximal delay for $c=5$ is $\tau_{0}=$ $5.1 \times 10^{-2}$. The estimation $c_{1}=0.1812$ is obtained in accordance with (11). Then the system (2), (6) is globally asymptotically stable with respect to $B_{27.5868}^{2 \tau}$ for any delay $\tau<\tau_{0}=5.1 \times 10^{-2}$.

It is easy to extend Lemma 3 for disturbed case.

Lemma 4 (Disturbed case) Let the system of linear matrix inequalities

$$
\left\{\begin{array}{l}
\kappa\left(A X+X A^{T}+b y+y^{T} b^{T}\right)+L_{1}+R_{1} \leq 0, \\
(1-\kappa)\left(A X+X A^{T}+b y+y^{T} b^{T}\right)+L_{2}+R_{2} \leq 0, \\
\iota X \geq A X+X A^{T} \geq-\iota X, \quad\left(\begin{array}{cc}
X & y^{T} \\
y & \zeta
\end{array}\right) \geq 0, \\
X H_{\mu}+H_{\mu} X \geq \eta X, \quad X>0, \quad L_{1} \geq \varpi_{1} X, \\
\varpi_{2} X \geq L_{2} \geq 0, \quad \varpi_{3} X \geq R_{2} \geq 0,
\end{array}\right.
$$


be feasible for $R_{1}=\left(\begin{array}{cc}0_{n-1} & 0 \\ 0 & r_{n n}\end{array}\right)$ and some $\mu \in(0,1]$, $\kappa \in(0,1), r_{n n}, \eta, \zeta, \iota, \varpi_{1}, \varpi_{2}, \varpi_{3} \in \mathbb{R}_{>}, X \in \mathbb{R}^{n \times n}$, $y \in \mathbb{R}^{1 \times n}$ and the inequality

$$
\begin{aligned}
& d^{T}(t, x(t)) D\left(V^{-1}(t)\right) R_{2}^{-1} D\left(V^{-1}(t)\right) d(t, x(t)) \leq \\
& \beta V^{-2 \mu}(t) x^{T}(t) D\left(V^{-1}(t)\right) P L_{2} P D\left(V^{-1}(t)\right) x(t)
\end{aligned}
$$

holds for $\beta \in(0,1)$ with $V(t)>c$. The system (2), (6) is globally asymptotically stable with respect to $B_{c / c_{1}}^{2 \tau}$ for any $c \in \mathbb{R}_{>}$if

$$
\begin{aligned}
& \tau<\left(\eta^{-1} \sqrt{l_{1}}\left(\iota+2 \sqrt{P_{n n} \zeta}+2 \sqrt{\varpi_{2} \varpi_{3}}\right)+\sqrt{l_{2}}+\right. \\
& \left.\left|k_{n}\right| \sqrt{\zeta}+\sqrt{\zeta \varpi_{2} \varpi_{3}}\right)^{-1} \sqrt{r_{n n} \varpi_{1}} c^{\mu},
\end{aligned}
$$

where $P=X^{-1}, k=y X^{-1}, l_{1}=\lambda_{\max }\left(X^{1 / 2}((1-\mu) I+\right.$ $\left.\left.H_{\mu}\right) k^{T} k\left((1-\mu) I+H_{\mu}\right) X^{1 / 2}\right), l_{2}=\lambda_{\max }\left(X^{1 / 2} A^{T} k^{T} k A X^{1 / 2}\right)$.

Note that if $\tau=0$ and the inequality (18) holds globally, then the origin is finite-time stable.

Proof. Rewrite for disturbance case the expression (15)

$$
\begin{aligned}
& |\dot{V}(x(t))|=\mid\left(V^{-1}(t) x^{T}(t) D\left(V^{-1}(t)\right)\left(H_{\mu} P+P H_{\mu}\right) \times\right. \\
& \left.D\left(V^{-1}(t)\right) x(t)\right)^{-1}\left(V^{-\mu}(t) x^{T}(t) D\left(V^{-1}(t)\right)\left(P A+A^{T} P\right) \times\right. \\
& D\left(V^{-1}(t)\right) x(t)+2 V^{-1}(t) V^{1-\mu}(t-\tau) x^{T}(t) D\left(V^{-1}(t)\right) P b k \times \\
& D\left(V^{-1}(t-\tau)\right) x(t-\tau)+ \\
& \left.2 x^{T}(t) D\left(V^{-1}(t)\right) P D\left(V^{-1}(t)\right) d(t, x(t))\right) \mid \leq \\
& \eta^{-1} V^{1-\mu}(t)\left(\iota+2 \gamma^{1-\mu} \sqrt{P_{n n} \zeta}\right)+ \\
& 2 \eta^{-1} V(t)\left|d^{T}(t, x(t)) D\left(V^{-1}(t)\right) P^{1 / 2}\right| \times \\
& \left|P^{1 / 2} D\left(V^{-1}(t)\right) x(t)\right| \leq \\
& \eta^{-1} V^{1-\mu}(t)\left(\iota+2 \gamma^{1-\mu} \sqrt{P_{n n} \zeta}+2 \sqrt{\varpi_{2} \varpi_{3} \beta}\right)
\end{aligned}
$$

and the expression (16)

$$
\begin{aligned}
& \left|\int_{t-\tau}^{t} \dot{u}(s) d s\right|= \\
& \int_{t-\tau}^{t} V^{-\mu}(s) \dot{V}(s) k\left((1-\mu) I+H_{\mu}\right) D\left(V^{-1}(s)\right) x(s) d s+ \\
& \int_{t-\tau}^{t} V^{1-2 \mu}(s) k A D\left(V^{-1}(s)\right) x(s)+ \\
& \int_{t-\tau}^{t} V^{-\mu}(s) V^{1-\mu}(s-\tau) k b k D\left(V^{-1}(s-\tau)\right) x(s-\tau) d s+ \\
& \int_{t-\tau}^{t} V^{1-\mu}(s) k D\left(V^{-1}(s)\right) d(s, x(s)) d s \mid \leq \\
& \left(\eta^{-1} \sqrt{l_{1}}\left(\iota+2 \gamma^{1-\mu} \sqrt{P_{n n} \zeta}+2 \sqrt{\varpi_{2} \varpi_{3} \beta}\right)+\right. \\
& \left.\sqrt{l_{2}}+\gamma^{1-\mu}\left|k_{n}\right| \sqrt{\zeta}\right)\left|\int_{t-\tau}^{t} V^{1-2 \mu}(s) d s\right|+ \\
& \int_{t-\tau}^{t} V^{1-\mu}(s)\left|k D\left(V^{-1}(s)\right) d(s, x(s))\right| d s \leq \\
& \tau c^{-\mu} \gamma^{1-\mu}\left(\eta^{-1} \sqrt{l_{1}}\left(\iota+2 \gamma^{1-\mu} \sqrt{P_{n n} \zeta}+2 \sqrt{\varpi_{2} \varpi_{3} \beta}\right)+\right. \\
& \left.\sqrt{l_{2}}+\gamma^{1-\mu}\left|k_{n}\right| \sqrt{\zeta}+\sqrt{\zeta \varpi_{2} \varpi_{3} \beta}\right) V^{1-\mu}(t) .
\end{aligned}
$$

According to [19] it can be shown that

$$
\begin{gathered}
\dot{V}(x(t))=\left(\begin{array}{c}
D\left(V^{-1}(t)\right) x(t) \\
D\left(V^{-1}(t)\right) d_{\tau}(t)
\end{array}\right)^{T} W_{1}\left(\begin{array}{c}
D\left(V^{-1}(t)\right) x(t) \\
D\left(V^{-1}(t)\right) d_{\tau}(t)
\end{array}\right)+ \\
\left(\begin{array}{c}
D\left(V^{-1}(t)\right) x(t) \\
D\left(V^{-1}(t)\right) d(t, x(t))
\end{array}\right)^{T} W_{2}\left(\begin{array}{c}
D\left(V^{-1}(t)\right) x(t) \\
D\left(V^{-1}(t)\right) d(t, x(t))
\end{array}\right)+ \\
+V^{\mu}(t) d_{\tau}^{T}(t) D\left(V^{-1}(t)\right) R_{1}^{-1} D\left(V^{-1}(t)\right) d_{\tau}(t)- \\
V(t)^{-\mu} x^{T}(t) D\left(V^{-1}(t)\right) P L_{1} P D\left(V^{-1}(t)\right) x(t)+ \\
+V^{\mu}(t) d^{T}(t, x(t)) D\left(V^{-1}(t)\right) R_{2}^{-1} D\left(V^{-1}(t)\right) d(t, x(t))- \\
V(t)^{-\mu} x^{T}(t) D\left(V^{-1}(t)\right) P L_{2} P D\left(V^{-1}(t)\right) x(t),
\end{gathered}
$$

where

$W_{1}=\left(\begin{array}{cc}V^{-\mu}(t) \kappa\left(P(A+b k)+(A+b k)^{T} P\right)+ & \\ P L_{1} P & P \\ P & -V^{\mu}(t) R_{1}^{-1}\end{array}\right)$,

are negative semidefinite. Then, taking into account (18) we obtain

$$
\begin{aligned}
& \dot{V} \leq V^{\mu}(t) d_{\tau}^{T}(t) D\left(V^{-1}(t)\right) R_{1}^{-1} D\left(V^{-1}(t)\right) d_{\tau}(t)- \\
& V(t)^{-\mu} x^{T}(t) D\left(V^{-1}(t)\right) P L_{1} P D\left(V^{-1}(t)\right) x(t)
\end{aligned}
$$

The remaining proof follows a line similar to Lemma 3 .

Remark 3 If the matching condition $d_{i}(t, x(t))=0$, $i=\overline{1, n-1}$ holds, then to relax the system (17) the matrix $R_{2}$ can be chosen as $R_{2}=\left(\begin{array}{cc}0_{n-1} & 0 \\ 0 & \bar{r}_{n n}\end{array}\right), \bar{r}_{n n} \in \mathbb{R}_{>}$.

Remark 4 Considering the case of sampled-data control realization for the system (1) by Lemma 3 (or Lemma 4) global attractiveness of a compact set containing the origin can be shown only. In [29] this issue was studied more precisely where global asymptotic stability of the origin is proven.

Example 2 Consider the system (2) for $n=3$ in the case of matched disturbances $d_{1}(t, x(t))=d_{2}(t, x(t))=0$, $d_{3}(t, x(t))=0.1 x_{3}^{0.4}$. Define the control (6) with the parameter $\mu=0.6$. The parameters of the control (6) were selected solving the LMIs (17) for $r_{n n}=0.807, \bar{r}_{n n}=0.1831, \kappa=0.8151, \eta=1$ :

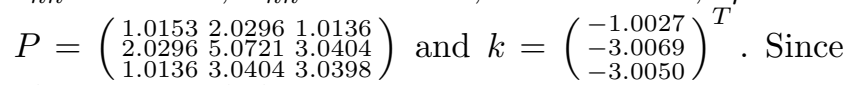
$d_{3}^{2} \leq 0.01\|x\|_{r}^{2-2 \mu}$ and $V(x) \geq c_{1}\|x\|_{r}$ it can be easily checked the inequality (18) holds. The estimation $c_{1}=0.218$ is obtained in accordance with (11). 
Then, according to Lemma 4 the system (2), (6) is globally asymptotically stable with respect to $B_{c / c_{1}}^{2 \tau}=$ $B_{13.7612}^{2 \tau}(c=3)$ for any delay $\tau \leq \tau_{0}=5.36 \times 10^{-2}$.

\subsection{Control providing asymptotic stability in presence of delay}

Using of Lemma 3 and Lemma 4 allows to design new hybrid stabilization algorithms, which preserve asymptotic stability of system trajectories.

Consider two implicit Lyapunov functions defined by

$$
\begin{aligned}
& Q_{1}(V, x)=x^{T} D_{\mu}\left(V^{-1}\right) P D_{\mu}\left(V^{-1}\right) x-1, \\
& Q_{2}(V, x)=x^{T} D_{\nu}\left(V^{-1}\right) P D_{\nu}\left(V^{-1}\right) x-1,
\end{aligned}
$$

where $P \in \mathbb{R}^{n \times n}, P>0, D_{\mu}(\lambda)=\operatorname{diag}\left\{\lambda^{1+(n-1) \mu}\right\}_{i=1}^{n}$ and $D_{\nu}(\lambda)=\operatorname{diag}\left\{\lambda^{1+(i-1) \nu}\right\}_{i=1}^{n}$. Denote $H_{\mu}=$ $\operatorname{diag}\{1+(n-i) \mu\}_{i=1}^{n}$ and $H_{\nu}=\operatorname{diag}\{1+(i-1) \nu\}_{i=1}^{n}$.

Then the following results can be obtained.

Theorem 4 (Disturbance-free case) Let for (2), $d(t, x(t))=0$ the system of linear matrix inequalities

$$
\left\{\begin{array}{l}
A X+X A^{T}+b y+y^{T} b^{T}+L_{\mu}+R_{\mu} \leq 0, \quad L_{\nu} \geq \varpi_{\nu} X, \\
A X+X A^{T}+b y+y^{T} b^{T}+L_{\nu}+R_{\nu} \leq 0, \quad L_{\mu} \geq \varpi_{\mu} X, \\
\iota X \geq A X+X A^{T} \geq-\iota X, \quad\left(\begin{array}{cc}
X & y^{T} \\
y & \zeta
\end{array}\right) \geq 0, \quad X>0 \\
X H_{\mu}+H_{\mu} X \geq \eta_{\mu} X, \quad X H_{\nu}+H_{\nu} X \geq \eta_{\nu} X
\end{array}\right.
$$

be feasible for $R_{\mu}=\left(\begin{array}{cc}0_{n-1} & 0 \\ 0 & r_{\mu}\end{array}\right), R_{\nu}=\left(\begin{array}{cc}0_{n-1} & 0 \\ 0 & r_{\nu}\end{array}\right)$ and some $\nu \in \mathbb{R}_{\geq}, \mu \in(0,1], \iota, \eta_{\mu}, \eta_{\nu}, r_{\mu}, r_{\nu}, \zeta, \varpi_{\mu}, \varpi_{\nu} \in$ $\mathbb{R}_{>}, X \in \mathbb{R}^{n \times n}, y \in \mathbb{R}^{1 \times n}$ and the control law $u$ is chosen in the form

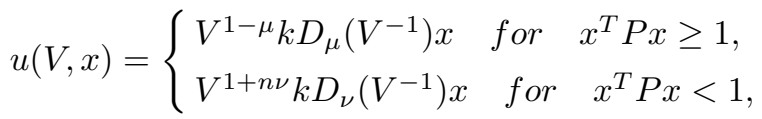

where $k=y X^{-1}, P=X^{-1}$ and $V$ is defined by

$$
V \in \mathbb{R}_{>}:\left\{\begin{array}{lll}
Q_{1}(V, x)=0 & \text { for } & x^{T} P x \geq 1 \\
Q_{2}(V, x)=0 & \text { for } & x^{T} P x<1
\end{array}\right.
$$

The system (2), (21) is globally asymptotically stable if

$$
\begin{gathered}
\tau<\min \left\{\frac{\sqrt{r_{\mu} \varpi_{\mu}}}{\eta_{\mu}^{-1} \sqrt{l_{1_{\mu}}}\left(\iota+2 \sqrt{P_{n n} \zeta}\right)+\sqrt{l_{2}}+\left|k_{n}\right| \sqrt{\zeta}},\right. \\
\left.\frac{\sqrt{r_{\nu} \varpi_{\nu}}}{\eta_{\nu}^{-1} \sqrt{l_{1_{\nu}}}\left(\iota+2 \sqrt{P_{n n} \zeta}\right)+\sqrt{l_{2}}+\left|k_{n}\right| \sqrt{\zeta}}\right\},
\end{gathered}
$$

where $l_{1_{\mu}}=\lambda_{\max }\left(X^{1 / 2}\left((1-\mu) I+H_{\mu}\right) k^{T} k\left((1-\mu) I+H_{\mu}\right) X^{1 / 2}\right)$, $l_{1_{\nu}}=\lambda_{\max }\left(X^{1 / 2}\left((1+\nu) I+H_{\nu}\right) k^{T} k\left((1+\nu) I+H_{\nu}\right) X^{1 / 2}\right)$ and $l_{2}=\lambda_{\max }\left(X^{1 / 2} A^{T} k^{T} k A X^{1 / 2}\right)$. Moreover, for any $\tau>0$ trajectories of the closed-loop system are globally bounded.

Proof. The formula (22) implicitly defines the Lyapunov function candidate, which can be prolonged by continuity to the origin $V(0)=0$. Note, that $x^{T} P x \leq 1 \Rightarrow$ $V(x) \leq 1$ and $x^{T} P x \geq 1 \Rightarrow V(x) \geq 1$.

Similarly to Lemma 3 it can be shown that the set $\Omega_{1}=$ $\left\{x \in c_{[-2 \tau, 0]}: V(x) \leq 1\right\}$ is asymptotically attractive.

For the function $Q_{2}(V, x)$ in the similar way as in the proof of Lemma 3 and according to [29] it can be shown, that $\dot{V}(x(t)) \leq-C V^{1+\nu}(x(t)), C \in \mathbb{R}_{>}$with $V(t) \geq 1 / \gamma$ if

$$
\begin{aligned}
& d_{\tau}^{T}(t) D_{\nu}\left(V^{-1}(t)\right) R_{\nu}^{-1} D_{\nu}\left(V^{-1}(t)\right) d_{\tau}(t) \leq \\
& \beta_{\nu} V^{2 \nu}(t) x^{T}(t) D_{\nu}\left(V^{-1}(t)\right) P L_{\nu} P D_{\nu}\left(V^{-1}(t)\right) x(t)
\end{aligned}
$$

for some $\beta_{\nu} \in(0,1)$.

The remaining proof follows a line similar to Lemma 3 to show the system is asymptotically stable in $\Omega_{1}$. Validity of Theorem 4 is thus concluded.

Theorem 4 proves very important advantage of nonlinear control (21). Once designed it remains robust (in ISS sense) with respect to any delay.

Example 3 Consider the system (2) for $n=3$ in the disturbance-free case. Define the control $u$ in the form (21) with the parameters $\mu=\nu=0.3$. The matrix $P>0$ and the vector $k$ are obtained from the system (20) with $r_{\mu}=1, r_{\nu}=2.5005$, $\eta_{\mu}=\eta_{\nu}=1.5, \varpi_{\mu}=\varpi_{\nu}=1: P=\left(\begin{array}{lll}0.3998 & 0.8005 & 0.3999 \\ 0.8005 & 2.0026 & 1.2014 \\ 0.3999 & 1.2014 & 1.2012\end{array}\right)$ and $k=\left(\begin{array}{l}-0.9998 \\ -3.0017 \\ -3.0017\end{array}\right)^{T}$. Obtained value of maximal delay is $\tau_{0}=\min \left\{2.68 \times 10^{-2}, 2.9 \times 10^{-2}\right\}=2.68 \times 10^{-2}$. According to Theorem 4 the system (2), (21) is globally asymptotically stable if $\tau<\tau_{0}=2.68 \times 10^{-2}$. Otherwise, similarly to Lemma 3 the system is converging to some compact set around the origin. The simulation results for $\tau=1.55 \times 10^{-2}$ are shown on Fig.1.

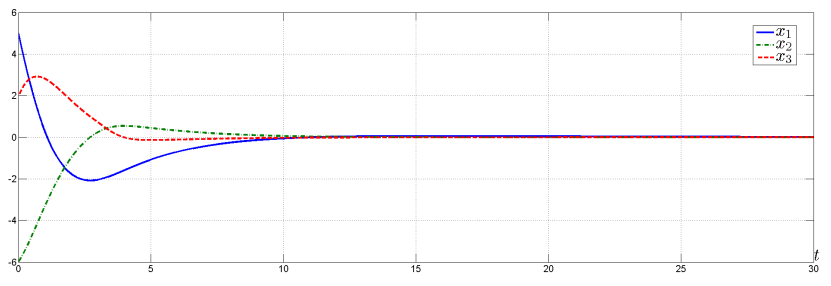

Fig. 1. The simulation results for the ILF control (21) 
Theorem 5 (Disturbed case) Let for (2) the system of linear matrix inequalities

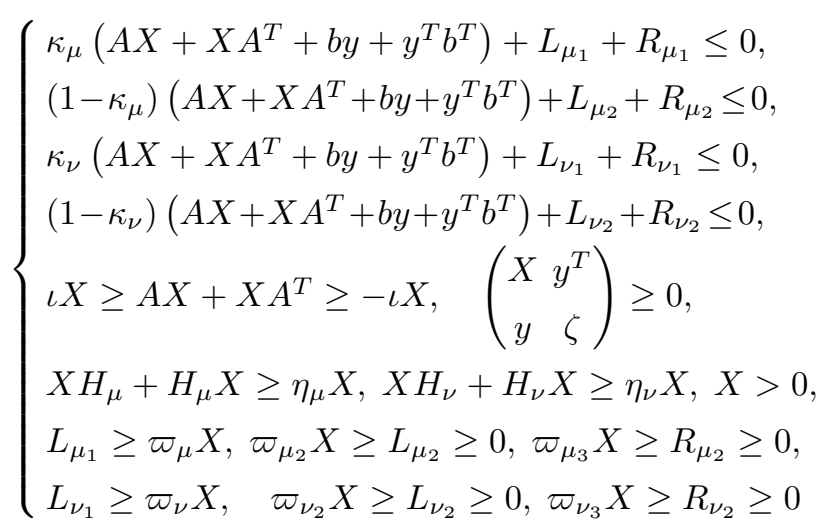

be feasible for $R_{\mu_{1}}=\left(\begin{array}{cc}0_{n-1} & 0 \\ 0 & r_{\mu}\end{array}\right), R_{\nu_{1}}=\left(\begin{array}{cc}0_{n-1} & 0 \\ 0 & r_{\nu}\end{array}\right)$ and some $\kappa_{\mu} \in(0,1), \kappa_{\nu} \in(0,1), \nu \in \mathbb{R}_{\geq}, \mu \in(0,1], \iota, \zeta$, $\eta_{\mu}, \eta_{\nu}, r_{\mu}, r_{\nu}, \varpi_{\mu}, \varpi_{\nu} \in \mathbb{R}_{>}, X \in \mathbb{R}^{n \times \bar{n}}, y \in \mathbb{R}^{1 \times n}$, the control law $u$ is chosen in the form (21) with $k=y X^{-1}$, $P=X^{-1}$ and the disturbance function $d(t, x)$ satisfies

$$
\begin{aligned}
& d^{T}(t, x) D_{\mu}\left(V^{-1}\right) R_{\mu_{2}}^{-1} D_{\mu}\left(V^{-1}\right) d(t, x) \leq \\
& \beta_{\mu} V^{-2 \mu} x^{T} D_{\mu}\left(V^{-1}\right) P L_{\mu_{2}} P D_{\mu}\left(V^{-1}\right) x \text { if } x^{T} P x \geq 1, \\
& d^{T}(t, x) D_{\nu}\left(V^{-1}\right) R_{\nu_{2}}^{-1} D_{\nu}\left(V^{-1}\right) d(t, x) \leq \\
& \beta_{\nu} V^{2 \nu} x^{T}(t) D_{\nu}\left(V^{-1}\right) P L_{\nu_{2}} P D_{\nu}\left(V^{-1}\right) x \text { if } x^{T} P x<1,
\end{aligned}
$$

where $\beta_{\mu} \in(0,1), \beta_{\nu} \in(0,1)$. Then the system (2), (21) is asymptotically stable if

$$
\begin{aligned}
& \tau<\min \left\{\left(\eta_{\mu}^{-1} \sqrt{l_{1_{\mu}}}\left(\iota+2 \sqrt{P_{n n} \zeta}+2 \sqrt{\varpi_{\mu_{2}} \varpi_{\mu_{3}}}\right)+\sqrt{l_{2}}+\right.\right. \\
& \left.\left|k_{n}\right| \sqrt{\zeta}+\sqrt{\zeta \varpi_{\mu_{2}} \varpi_{\mu_{3}}}\right)^{-1} \sqrt{r_{\mu} \varpi_{\mu}}, \\
& \left(\eta_{\nu}^{-1} \sqrt{l_{1_{\nu}}}\left(\iota+2 \sqrt{P_{n n} \zeta}+2 \sqrt{\varpi_{\nu_{2}} \varpi_{\nu_{3}}}\right)+\sqrt{l_{2}}+\right. \\
& \left.\left.\left|k_{n}\right| \sqrt{\zeta}+\sqrt{\zeta \varpi_{\nu_{2}} \varpi_{\nu_{3}}}\right)^{-1} \sqrt{r_{\nu} \varpi_{\nu}}\right\}
\end{aligned}
$$

where $l_{1_{\mu}}=\lambda_{\max }\left(X^{1 / 2}\left((1-\mu) I+H_{\mu}\right) k^{T} k\left((1-\mu) I+H_{\mu}\right) X^{1 / 2}\right)$, $l_{1_{\nu}}=\lambda_{\max }\left(X^{1 / 2}\left((1+\nu) I+H_{\nu}\right) k^{T} k\left((1+\nu) I+H_{\nu}\right) X^{1 / 2}\right)$ and $l_{2}=\lambda_{\max }\left(X^{1 / 2} A^{T} k^{T} k A X^{1 / 2}\right)$. Moreover, for any $\tau>0$ trajectories of the closed-loop system are globally bounded.

The proof is omitted since it repeats the arguments given in the proofs of Lemma 4 and Theorem 4.

Example 4 Consider the system (2) for $n=3$ with disturbances $d_{1}(t, x(t))=d_{2}(t, x(t))=0, d_{3}(t, x(t))=$ $0.1 \sin \left(x_{1}^{1.2}(t)\right)$. Define the control $u(t)$ in the form $(21)$ with the parameters $\mu=1, \nu=0.1$. The matrix $P>0$ and the vector $k$ are obtained from the system (24) with
$R_{\mu_{2}}=\left(\begin{array}{cc}0_{2} & 0 \\ 0 & 0.1005\end{array}\right), R_{\nu_{2}}=\left(\begin{array}{cc}0_{2} & 0 \\ 0 & 1.0751\end{array}\right), r_{\mu}=0.9045, r_{\nu}=$ 1.4251, $\eta_{\mu}=1.8, \eta_{\nu}=1.5, \varpi_{\mu}=\varpi_{\nu}=1, \kappa_{\mu}=0.9, \kappa_{\nu}=0.57$ : $P=\left(\begin{array}{lll}1.3709 & 2.2166 & 0.5195 \\ 2.2166 & 4.8753 & 1.4829 \\ 0.5195 & 1.4829 & 1.4819\end{array}\right)$ and $k=\left(\begin{array}{l}-1.9695 \\ -4.9930 \\ -3.3534\end{array}\right)^{T}$. Note, that for the function $Q_{2}(V, x)$ the Lyapunov function is homogeneous of degree 1 with $r=(1,1+\nu, \ldots, 1+(n-$ 1) $\nu)^{T}$. Then, we can use the following estimation of the parameter $c_{1_{\nu}} \in \mathbb{R}_{>}: c_{1_{\nu}}\|x\|_{r} \leq V$ :

$$
c_{1_{\nu}}=\min \left\{\frac{\lambda_{\min }^{\frac{1}{2+2(n-1) \nu}}(P)}{n^{\frac{1}{\rho}}}, \frac{\lambda_{\min }^{\frac{1}{2}}(P)}{n^{\frac{1}{\rho}}}\right\}=0.2031 .
$$

Since $\sin \left(x_{1}\right) \leq\left|x_{1}\right| \Rightarrow \sin ^{2}\left(x_{1}^{1.2}\right) \leq|x|_{r_{\nu}}^{2.4}$ it can be easily shown that inequalities (25) hold.

Obtained value of maximal delay is $\tau_{0}=\min \{2.24 \times$ $\left.10^{-2}, 1.83 \times 10^{-2}\right\}=1.83 \times 10^{-2}$. According to Theorem 5 the system $(2),(21)$ is globally asymptotically stable if $\tau<\tau_{0}=1.83 \times 10^{-2}$. Otherwise, similarly to the result of Lemma 4 the system is converging to some compact set around the origin. The simulation results for constant delay $\tau=10^{-2}$ are shown on Fig.2.

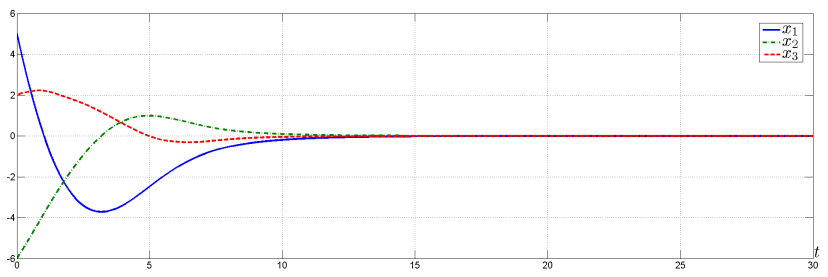

Fig. 2. The simulation results for the ILF control (21)

Let us compare the results with sliding-mode control law presented in [31], which is closest analogue since

- it also implies ultimate boundedness of the closed-loop system;

- in the case $\mu=1$ the control law (6) realizes a high order sliding mode control rejecting matched bounded uncertainties and disturbances;

- the parameter tuning procedure is in the form of LMIs.

The benefits and advantages of the designed control laws are as follows:

- the control law (21) is continuous; the control law (6) is continuous for $\mu \in(0,1)$ and continuous outside the origin for the case $\mu=1$;

- the control laws are robust with respect to both matched and unmatched disturbances;

- the control law (21) provides asymptotic stability of the origin for sufficiently small input delay;

- changing the parameter $\mu$ one can change restrictions on the disturbance function $d(t, x)$ and the convergence region. 


\section{Conclusion}

The paper presents ILF-based control algorithms for systems with the delay in control (and/or measurement) channel, i.e. teleoperated and networked systems. Obtained nonlinear control algorithms preserve asymptotic stability for small delays $0<\tau<\tau_{0}<\infty$ and global asymptotic stability with respect to a compact set containing the origin for any $\tau \geq \tau_{0}$. The results can be used for the cases of a constant and fast-varying delays. Efficiency of the proposed control algorithms is demonstrated on numerical examples. There are a lot of topics for future research. For example, the presented results can be extended for linear MIMO systems using generalized homogenising stabilization algorithm presented in [30]. Other directions are relaxation of restrictions, extension to output control case, study of transient performances, etc.

\section{References}

[1] K. Zimenko, D. Efimov, A. Polyakov, A. Kremlev Control of Systems with Arbitrary Bounded Input Delay Using Implicit Lyapunov Function Technique. European Control Conference (ECC 2018), pages 1548-1553, 2018.

[2] J.-P. Richard. Time-delay systems: an overview of some recent advances and open problems. Automatica, vol. 39(10), pages 1667-1694, 2003

[3] L. Bushnell. Editorial: Networks and control. IEEE Control System Magazine, vol. 21(1), pages 22-99, 2001.

[4] C.-C. Hua, X.P. Liu. Editorial: Delay-Dependent Stability Criteria of Teleoperation Systems With Asymmetric TimeVarying Delays. IEEE Transactions on Robotics, vol. 26(5), pages 925-932, 2010.

[5] E. Fridman. Tutorial on Lyapunov-based methods for timedelay systems. European Journal of Control, vol. 20(6), pages 271-283, 2014.

[6] T. Slama, A. Trevisani, D. Aubry, R. Oboe, F. Kratz. Experimental Analysis of an Internet-Based Bilateral Teleoperation System With Motion and Force Scaling Using a Model Predictive Controller. IEEE Transactions on Industrial Electronics, vol. 55(9), pages 3290-3299, 2008.

[7] S. Hirche, M. Buss. Human-Oriented Control for Haptic Teleoperation. Proceedings of the IEEE, vol. 100(3), pages 623-647, 2012.

[8] E. Fridman. Introduction to Time-Delay Systems: Analysis and Control. Birkhuser, Basel, 2014.

[9] A. Pyrkin, A. Bobtsov. Adaptive Controller for Linear System with Input Delay and Output Disturbance. IEEE Transactions on Automatic Control, vol. 61(12), pages. 42294234, 2016.

[10] L. Mirkin, G. Tadmor. $H_{\infty}$ control of systems with I/O delay: A review of some problem-oriented methods. IMA Journal of Mathematical Control and Information, vol. 19(12), pages. 185-200, 2002.

[11] F. Gouaisbaut, W. Perruquetti, Y. Orlov, J-P. Richard. Sliding mode controller design for linear time delay systems. European Control Conference (ECC), pages 3780-3785, 1999.

[12] L. Fridman, E. Fridman, E. Shustin. Steady modes in relay control systems with delay. In Sliding mode control in engineering. New York: Marcel Dekker Inc., 2002.
[13] Yu. Mikheev, V. Sobolev, E. Fridman. Asymptotic analysis of digital control systems. Autom. Remote Control, vol. 49, pages $1175-1180,1988$.

[14] A. Isidori. Nonlinear Control Systems II. Springer-Verlag London, 293 p., 1999.

[15] A. Filippov. Differential equations with discontinuous righthand sides. Dordrecht, Kluwer, 1988.

[16] E. Cruz-Zavala, J.A. Moreno, L. Fridman. Uniform SecondOrder Sliding Mode Observer for mechanical systems. 11th International Workshop on Variable Structure Systems, 2010.

[17] M. Livne, A. Levant. Accuracy of disturbed homogeneous sliding modes. the 13th Scientific Workshop VSS13, 2014.

[18] A. Levant. Homogeneity of differential inclusions: Application to sliding modes. European Control Conference (ECC 2015), pages 2458-2463, 2015.

[19] A. Polyakov, D. Efimov, W. Perruquetti. Robust Stabilization of MIMO Systems in Finite/Fixed Time. Int. J. Robust. Nonlinear Control, vol. 26(1), pages 69-90, 2016.

[20] K. Gu, K.L. Kharitonov, J. Chen. Stability of Time-Delay Systems. Control Engineering, Birkhauser, Boston, 2003.

[21] D. Efimov, W. Perruquetti, J.-P. Richard. Development of homogeneity concept for time-delay systems. SIAM J. Control Optim., vol. 52(3), pages 1547-1566, 2014.

[22] V.I. Korobov. A general approach to synthesis problem. Doklady Academii Nauk SSSR, vol. 248, pages 1051-1063, 1979.

[23] J. Adamy, A. Flemming. Soft variable-structure controls: a survey. Automatica, vol. 40, pages 1821-1844, 2004.

[24] D. Efimov, W. Perruquetti. Homogeneity for time-delay systems. In Proc. IFAC WC 2011, Milan, 2011.

[25] A. Bacciotti, L. Rosier. Lyapunov Functions and Stability in Control Theory. Springer, 237 p., 2005.

[26] D. Efimov, A. Polyakov, W. Perruquetti, and J.-P. Richard. Weighted homogeneity for time-delay systems: Finite-time and independent of delay stability. IEEE Trans. Automatic Control, vol. 61(1), pages 210-215, 2016.

[27] V.B. Kolmanovsky and V.R. Nosov. Stability of functional differential equations. CA: Academic, San Diego, 1986.

[28] K. Zimenko, D. Efimov, A. Polyakov, and W. Perruquetti. A note on delay robustness for homogeneous systems with negative degree. Automatica, vol. 79, pages 178-184, 2017.

[29] A. Polyakov, D. Efimov, and W. Perruquetti. Finite-time and fixed-time stabilization: Implicit Lyapunov function approach. Automatica, vol. 51, pages 332-340, 2015.

[30] K. Zimenko, A. Polyakov, D. Efimov, W. Perruquetti. Generalized Feedback Homogenization and Stabilization of Linear MIMO Systems. European Control Conference (ECC 2018), pages 1987-1991, 2018.

[31] X. Han, E. Fridman, S.K. Spurgeon. Sliding mode control in the presence of input delay: A singular perturbation approach. Automatica, vol. 48(8), pages 1904-1912, 2012.

[32] L. Belkoura, Y. Orlov. Identifiability analysis of linear delaydifferential systems. IMA Journal of Mathematical Control and Information, vol. 19(1-2), pages 73-81, 2002. 